Article

\title{
Altered Self-Observations, Unclear Risk Perceptions and Changes in Relational Everyday Life: A Qualitative Study of Psychosocial Life with Diabetes during the COVID-19 Lockdown
}

\author{
Dan Grabowski *, Julie Meldgaard and Morten Hulvej Rod \\ Steno Diabetes Center Copenhagen, Niels Steensens Vej 6, 2820 Gentofte, Denmark; \\ julie.meldgaard.petersen@regionh.dk (J.M.); morten.hulvej.rod@regionh.dk (M.H.R.) \\ * Correspondence: dan.grabowski@regionh.dk
}

Received: 18 July 2020; Accepted: 30 August 2020; Published: 1 September 2020

check for updates

\begin{abstract}
When the Danish society went into COVID-19 lockdown, it dramatically changed the conditions for living with a chronic disease like diabetes. The present article highlights the psychosocial effects of this change. The dataset consists of 20 semi-structured online interviews with people with diabetes. The data were analyzed using radical hermeneutics and interpreted using Luhmann's operative constructivist systems theory. The analysis produced three main themes: (1) people with diabetes experience altered self-observations-mainly due to society labelling them as vulnerable, (2) people with diabetes have unclear risk perceptions due to lack of concrete knowledge about the association between COVID-19 and diabetes, and (3) changes in conditions for maintaining and creating meaningful relations have a significant impact on everyday life with diabetes. These findings have important implications for risk communication. People respond in a multitude of ways to communications issued by health authorities and with close relations, and their meaning-making is shaped by, and shapes, their self-observations, risk perceptions and relational environments. This calls for more targeted communication strategies as well as increased use of peer support; the goal being to help people create meaning in their own environments.
\end{abstract}

Keywords: COVID-19; diabetes; psychosocial effects; self-observations; risk perceptions; social relations; systems theory; qualitative research

\section{Introduction}

The spread of the 2019 novel coronavirus was declared a global pandemic by the World Health Organization on 11 March 2020 [1]. The virus most often only causes mild symptoms similar to those of a typical influenza, but in some people, it can cause severe respiratory infections and multi-organ failure [2]. These severe symptoms are more likely to occur in specific high-risk groups. The designation of such high-risk groups has evolved during the course of the pandemic and has differed across countries. On 11 March, the Danish Health Authority declared people with poorly regulated diabetes (all types) as a group at high risk for becoming seriously ill if infected with the virus [3].

Studies investigating the prevalence of diabetes (all types) in COVID-19 patients have found it to be around $10 \%[4,5]$, but it remains unclear whether people with diabetes are more susceptible to COVID-19 [6,7]. However, numerous studies have shown an increased risk of severe COVID-19 and mortality in individuals with diabetes [4,6-12], as well as higher rates of hospitalization due to COVID-19 [13,14]. Thus, individuals with diabetes have been identified as having worse COVID-19 outcomes, particularly those with poor glycemic control $[15,16]$. 
This information on the association between diabetes and COVID-19 was not, however, available during the early stages of the pandemic due to the novelty of the virus [17]. As a result of this uncertainty and lack of evidence-based knowledge about the virus, the information and messages from health authorities, and therefore also from the media, have been mixed and unclear, with different predictions and inconsistent risk communication [18]. This also applies to Denmark, where information from health authorities has varied over the course of the pandemic: Initially, only individuals with poorly regulated diabetes were said to be at risk of severe COVID-19, but later all people with diabetes, regardless of glycemic control and diabetes type, were categorized as a risk group [3].

Just as in many other countries, the Danish government enforced a lockdown to avoid critical spreading of the 2019 novel coronavirus [19]. On 12 March, Denmark closed all schools and childcare institutions, sent home public sector employees with non-critical roles and banned gatherings of more than 10 people.

A pandemic-induced lockdown can be expected to reveal social, psychological and underlying philosophical issues that will have a lasting impact on societies and individuals [20]. To study how a complex societal development such as the lockdown affects the self-observations and perceptions of communicated risk among people living with a chronic disease, we need to apply complexity-oriented social theory that enables us to look closely at the interrelatedness of the individual and the social. In the present study, we look through the lens of Niklas Luhmann's operative constructivist systems theory [21-23]. The objective of the present paper is to study how COVID-19 lockdown affects psychosocial life with diabetes. By applying significant concepts from Luhmann's systems theory, and furthermore by analyzing using radical hermeneutics [24], we examine how people with diabetes observe themselves in relation to other people, various social systems in their environment as well as the lockdown as an important societal phenomenon. Applying a qualitative approach with a distinct focus on individuals' thoughts, perceptions, and views enable us to understand how people with diabetes create meaning out of the complex multitude of communications concerning the consequences of the 2019 novel coronavirus.

\section{Materials and Methods}

\subsection{Empirical Data}

The dataset consists of 20 semi-structured individual interviews with people with diabetes (see Table 1 for characteristics). Due to the lockdown and the need for social distancing, the interviews were conducted and recorded online using a secure communication platform with the video function disabled. We decided to disable the video function because especially the older interviewees might be fairly unfamiliar with video conferences and could find this to be intimidating.

Table 1. Study population characteristics.

\begin{tabular}{cc}
\hline Participants & $(\boldsymbol{n}=\mathbf{2 0})$ \\
\hline Mean age in years, [range] & $51.7[20-75]$ \\
Gender, female & 10 \\
Diabetes type & \\
Type 1 & 8 \\
Type 2 & 11 \\
LADA * & 1 \\
Mean diabetes duration in years [range] & $14.9[0-48]$ \\
\hline \multicolumn{2}{c}{ * Relatively rare form of diabetes. }
\end{tabular}

The relatively simple interview guide consisted of four main themes: (1) diabetes management (in general and during lockdown): with questions focusing on the day-to-day issues regarding medication, exercise and diet, (2) quality of life (in general and during lockdown): with questions focusing on perceptions of general well-being, (3) perception of being at risk (in general and during 
lockdown): with questions focusing on health and risk communication and the specific relation between diabetes and COVID-19, and (4) relations and support (in general and during lockdown): with questions focusing on interactions with friends, family members, colleagues and healthcare professionals.

The participants were recruited through the user panels at Steno Diabetes Center Copenhagen and the Danish Diabetes Association. The user panels consist of people with diabetes who have volunteered to share information about their lives with diabetes. The panels include people from all parts of Denmark with type 1 diabetes (T1D) and type 2 diabetes (T2D, LADA, gestational diabetes and other rarer types of diabetes. Thus, the panels represent people with diabetes who are treated in different primary and/or secondary care settings across Denmark. The panel members $(n=2430)$ received the invitation to participate in an interview via an e-mail newsletter sent out to all panel members. The interested panel members then contacted the research team by e-mail or telephone.

We decided to be open to recruit participants with T1Dand T2D so that we could study potentially different reactions to the lockdown. During one interview we discovered that the interviewee actually had LADA, which is a relatively rare form of diabetes that has similarities with both T1D and T2D [25]. We decided to keep this interview in the dataset even though it did not contribute any insights into any specificities regarding living with LADA during lockdown. It did, however, contribute to the overall findings on living with diabetes during lockdown. Thus, in the present paper, we focus on persons with T1D and T2D

After each interview, the researchers discussed and systematically wrote down all the overarching themes that were touched upon in the particular interview. After 15 interviews, the research group used this overview to discuss the level of saturation and decided to conduct five additional interviews, because the interviews, especially with people with T2D, lacked information power. The recruitment procedure was not changed for the next five interviews that included three people with T2D and two people with T1D. When a total of 20 interviews had been conducted, we concluded that no new overarching new themes had emerged in the two latest interviews, meaning that the level of saturation and ensuing information power in the data were acceptable [26]. No changes were made in the interview guide at any point in the data collection period. All interviews were conducted over the course of two weeks and the average length was $40 \mathrm{~min}$. None of the researchers had any prior relationship with any of the interviewees.

After collecting the full dataset, the interviews were transcribed verbatim, iteratively analyzed in Danish and then categorized using radical hermeneutics, which are a set of guidelines for content analysis that, as a combination of hermeneutics and constructivism, manages to simultaneously be empirically founded and theoretically complex [24]. Radical hermeneutics focuses on keeping a perpetual balance between theory, method and data by acknowledging how all of these elements influence each other in an interconnected process. The use of radical hermeneutics also entails constant alternation between analyzing and interpreting, which means it is necessary to present interpretive aspects while presenting the results.

Radical hermeneutics is a validated methodology consisting of three steps of data analysis. The first step involves reading the data with a view to observing specifically selected differences in them. This observation in itself constitutes an interpretation rather than a description, and its task is to reduce the complexity of the data. Elements within the scope of the differences selected by the interpreter are extracted from the data. The second step involves making these elements the subject of interpretation as an observation of the differences employed. The third step involves interpreting the sum of these differences [24].

In the present analysis, this approach meant that the analytical process was developed in several steps as we delved deeper into the data. The first step focused on extracting issues of direct relevance to diabetes and the COVID-19 lockdown from the empirical data. The second step involved analyzing and interpreting the extracted data using the theoretical background described below, and this step revealed the three main themes presented in the results section. The final step is then a separate interpretation of the data within each category-materializing as the findings presented for each of the 
three themes. Working with radical hermeneutics includes the awareness that the role of the researcher is that of an observer. This underlines how any research depends on an awareness of how all the observations were made. This is exactly what radical hermeneutics and the use of guiding differences provide - a way to continually employ a high level of reflexivity in all aspects of the study.

The study was approved by the Danish Data Protection Agency (P-2020-271) and carried out in accordance with the Declaration of Helsinki. According to Danish legislation, interview studies require no approval from an ethics committee. All participants gave their informed consent based on thorough explanations of the purpose of the study. All participants were explained that they had the right to not answer any question and that they could stop the interview at any time if they were not comfortable with the situation. Furthermore, they were given details of whom to contact for answers to questions about the research and the research subject's rights.

\subsection{Theory}

In the present study, we adopt the theoretical position that every person observing his or her own unique environment is observing something slightly different from what everyone else is observing. This is important to bear in mind in relation to COVID-19 risk communication, as every person will be observing this in a slightly different way, and the messages will therefore mean something different in the context of different environments [23].

Luhmann's systems theory is, in essence, a grand theory about communication and the individual person's observation and meaning-making of this communication. The theory introduces a distinction between psychological and social systems, which is an important aspect of our present approach to studying life with diabetes during lockdown. What Luhmann called psychological systems, in fact, constitute the consciousness of persons, individuals or subjects, and for Luhmann, social systems can be either interactions, organizations or societies [23].

The consciousness of psychological systems and the communication of social systems do not have direct access to each other. They basically speak different languages, and this means that the psychological systems cannot communicate, but are instead restricted to observing the communicative social systems. The systems are closed and autopoietic, which means that each system continually self-interprets and self-reflects on the basis of its own contents. This inability to fully understand the other kind of system is Luhmann's way of addressing the classical sociological issue of the co-evolution of the social and the individual [22].

The notion that the systems are closed means that the consciousness that continually recreates the way we (as psychological systems) interpret and understand ourselves, in the context of our own unique environment, always comes from within the system itself. Consciousness never comes from the outside, because we never acquire consciousness from other psychological or social systems. We are therefore closed in terms of this operational element. It is this self-reference that enables systems to continually distinguish themselves from their environment. The concept of self-reference is therefore essential in understanding how each system is able to maintain its own horizon of meaning despite the complexity of the environment [21].

The psychological system continues to produce the consciousness needed to maintain a sense of meaning, while at the same time observing and trying to understand the environment. In this sense, we arrive at a paradox: While the systems are closed in terms of their self-reference, they are in fact at the same time open with regard to observing the environment. The concept of observation is therefore essential to how systems evolve and change. To actively try to understand and make sense of the environment, the psychological system needs to continually recreate its own self-understanding and sense of meaning. It is on the basis of this self-understanding and sense of meaning that the actual observations are made [22].

For the social systems to influence the psychological systems, and vice versa, the systems need to create noise or distortion in order for the other system to observe and try to make sense of it all. For the psychological system of a person with diabetes to be influenced by the COVID-19 risk communication, 
it has to see it as relevant to observe-and observing it has to make sense for the self-reproducing meaning-making processes within the psychological system itself. Observing communication from different social systems represents a high level of complexity, as the individual psychological system is exposed to numerous social systems on a daily basis [23].

The basic operation of observing is making a distinction. When a system observes something, it is automatically not observing something else. Rather, it is not observing the rest of the environment. When people with diabetes are observing anything health-related in any given setting, they are automatically not observing something else in relation to the health-related issue and/or the setting. By analyzing how, where and why these distinctions are made, we can analytically sharpen our focus on why people observe what they observe and, perhaps more importantly, why they do not observe what they do not observe [22].

To understand how systems navigate in the complex environment of possible observations, it is necessary to move on from the concept of observations to what Luhmann called expectational structures. By ascribing expected meaning to different contexts or relations, a person will acquire a sense of where he or she will be able to observe meaning in relation to him- or herself or in relation to any given prior observation [23].

\section{Results}

The analysis produced three main themes. One overarching finding within all three themes concerns the significant differences between how people with T1D and those with T2D experience themselves, their supportive relations and their diabetes in relation to COVID-19 during the lockdown. This overarching finding is present in all of the following themes, which can be seen as different ways of highlighting and interpreting this difference from various angles and perspectives.

In the presentation of the themes, we will not discuss the theoretical elements explicitly, as we will do that in the discussion. All the themes and the elements within the themes did, however, emerge during the analysis and are therefore linked to complexities of communication, social and psychological systems, meaning-making, observational structures and/or expectational structures.

The presented quotes have been translated from Danish to English.

\subsection{Altered Self-Observations}

Most of the participants had experienced having to change their view of themselves during the lockdown. The reasons for these changes were, however, manifold. The young female interviewee below, with T1D, found it difficult to suddenly be classified as 'vulnerable':

"I find it difficult to label myself as vulnerable! I normally see myself as a strong person, but I really do need people to take special precautions for me right now. It's hard to relate to myself in all this, when they call us vulnerable or as part of vulnerable groups. They keep saying that Corona isn't dangerous unless you're already ill and vulnerable, so I guess that's me now? Ill and vulnerable?"

(i2, female, 26y, T1D)

Finding yourself suddenly belonging to a vulnerable group has had a severe impact on all the interviewees with T1D. Many of them had seen themselves as strong independent people who were in control of their disease and their life in general, and most of them had successfully constructed a meaningful self-understanding of themselves based on having T1D. This self-understanding had, in many cases, been severely threatened by this new situation, in which diabetes was suddenly observed by everyone in their environment as a source of a specific vulnerability. Their own observations of the social system observing them as vulnerable basically had forced them to re-create their sense of meaning and, thereby, to change their ways of observing as well as their expectational structures regarding themselves and their environment.

Being in the vulnerable category did not merely entail having an unwanted, ill-fitting label put on you because these people were themselves very worried about the coronavirus-so they also labelled 
themselves as being in need of special attention. This created an observational paradox: Most of them observed themselves as being at risk, while they at the same time found it difficult to be externally observed as vulnerable. This was especially difficult because internal and external observations will always be strongly interdependent owing to the ongoing creation and re-creation of meaning. They might have no problem identifying with the disease and the increased risks, but the ensuing label was disturbing for these people because many of them had done considerable identity work that would allow them to identify positively with their disease:

"I do want all the love and people being considerate and showing me that they're taking care of me and that they know I need to be treated with caution. But I don't want to be "vulnerable" I want to be taken seriously—but without the label" (i2, female, 26y, T1D)

All of the interviewees with T1D generally identified quite strongly with their disease, and this seems to be the reason why the vulnerable label caused considerable confusion and identification problems. For the interviewees with T2D, the situation was more or less the opposite. Most of them did not identify as someone with a serious disease, and therefore many of them did not identify with belonging to a risk group either.

"I do not feel that I'm a weakened person in any way because I have this diabetes 2. I might be one, but I don't feel it ... Well, I got this feeling of 'oh my god, am I really a sick and weak person.' It stays in me for a short while but then it's out again ... But I do think a little bit about it ... but am I really that bad? Am I really in a risk group? I don't feel that I am, but am I? I'm never sick and I'm not sick more often after getting diabetes." (i19, male, 54y, T2D)

In our study, the interviewees with T2D did not generally observe themselves as people with a serious disease, and therefore they did not normally relate to being vulnerable or belonging to a risk group either. Most of them had no problem discarding the social systems observing them as being at risk, and therefore this label did not affect how they created meaning and self-understanding. They did take the lockdown restrictions seriously, but not because of their diabetes.

"I guess it's because I don't have any real problems with my diabetes. Other people might have, but I must admit, that I'm not the kind of person who takes it that seriously. I take it as it is and it's not like I'm dying because some doctor told me I have this disease. I try to live completely normal." (i11, female, 71y, T2D)

The interviewees with T2D tended to adopt this approach to diabetes and the risk of COVID-19. They understood themselves as not needing to take their diabetes particularly seriously, and they, therefore, did not relate to being in a particular risk group.

Creating meaningful self-understandings and/or changing the expectational structures for self-observations is often closely related to suddenly feeling different from others. Several of the interviewees in our study told us that the precautions they had to take during the lockdown made them feel different:

"When I started wearing the mask people looked at me like: 'Oh my, she's hysterical' Everyone thought I was overreacting when I was the only one in the mall with a mask on. I felt like I needed a big sign on my back reading "I' $m$ the one who is sick" (i18, female, 44y, T1D)

A different and significantly more positive way of observing yourself differently in the lockdown context is as someone who is actually handling it all pretty well. Some of the interviewees reported finding strength and meaning in observing themselves as able to handle difficult personal and societal changes very well:

"This might sound silly, but I actually think that I'm handling all of this pretty well. I think I need to give myself credit for this and to have a bit more self-confidence. I'm pretty damn good at this." (i15, female, 23y, T1D) 
This young woman compared herself to her peers in various diabetes peer-to-peer Facebook groups; she had observed a general frustration and fear among the other members. Observing this construction of meaning in a social system of peers had caused her to realize that she stood out from the group, and she then changed her expectational structures and group affiliation accordingly. These changes in her observation of her environment then significantly changed the way she observed herself as competent to cope with COVID-19 fear as well as the lockdown challenges.

\subsection{Unclear Risk Perceptions}

How the individual with diabetes perceives the combined risk of diabetes and COVID-19 is closely related to how that person perceives diabetes as an integrated part of everyday life and of the above-presented self-understanding.

The pressure of not fully understanding the actual risk was almost unbearable for several of the interviewees with T1D, all of whom tended to identify strongly with their disease. When faced with complex changes in the environment or a general rise in the level of complexity, every system will react by trying to observe identifiable structures and create a sense of meaning. In many cases, the lack of coherent knowledge and firm assessments of the COVID-19 risk for people with diabetes made this creation of meaning difficult.

"I can't not know anything. The pressure of not knowing the risk is greater than the fear of contracting it some days. You just get a $f X X X$-it-all kind of feeling and end up giving up on yourself and your health-and I think that's the worst that can happen. That's the kind of pressure you get from not understanding anything." (i6, female, 27y, T1D)

If something is perceived as very risky, thereby threatening the way you normally create a meaningful sense of yourself, it can significantly change self-understandings and structures of meaningful observations.

This suddenly emerged new complexity was also evident on the level of everyday diabetes management. The interviewees (again, especially those with T1D) could not seem to regulate their blood glucose in the way they had before the lockdown-due to changes in everyday routines. This caused more hands-on worries:

"Yes, of course, I'm a little worried. It's been difficult to regulate myself. I have a feeling that the more unstable your blood glucose is the worse your immune system is-and that makes me think that the virus might find an easier way in with someone who is poorly regulated. So that's the reason I've been more scared or nervous-I've been poorly regulated" (i7, male, 20y, T1D)

Thus, the interviewees did not only have to come to terms with a virus that puts them at a higher risk than the rest of the population. They also had to try to navigate their daily disease regulation through unknown territory, while trying to constantly reduce the complexity of the whole situation affecting how they saw themselves, how they observed their disease and how they made sense of their whole environment, as defined by societal systems now difficult to observe meaningfully.

This apparent state of confusion among the interviewees with T1D regarding risk seemed to be further enforced by the frustrations shared in Facebook groups:

"... and I hear from others that there are several with T1D who get sick and contact their doctor because they're also poorly regulated and therefore in the official risk group. But then the doctor refuses them a corona test and that's just not OK... They are just being told that they're young and fit and therefore not at risk. We're just not being taken seriously" (i2, female, 26y, T1D)

This shared identity of belonging to a risk group seemed to be both good and bad in relation to risk perceptions and being able to positively relate to diabetes. There was a distinct feeling of being ignored as a risk group or even left out of the closed-down society, which contributed to negative expectation structures that seemed to then turn into negative views on diabetes identification and 
negative self-understandings. On the other hand, it did seem to provide common ground in a time of solitude and potential loneliness.

"It's just really nice to write with and talk to my girlfriends who also have diabetes, because they feel the exact same way. I mean, everyone else understands absolutely nothing about how we feel right now. No one informs us about anything, and we're all gaining weight 'cause we've eaten too much cake like the rest of Denmark. It's just really nice to feel understood" (i2, female, 26y, T1D)

The situation was, once again, quite different for most of the interviewees with T2D. In most cases, they did not observe themselves as being at an increased risk, and several of them did not acknowledge any noteworthy connection between T2D and COVID-19:

"From my perspective, I just can't see what the two things have got to do with each other... I mean, I do understand that if you're a diabetes patient who eats four bags of crisps every day and weigh three times what you're supposed to and never leaves the house and everything else you can do wrong, then 'yes' maybe your world will fall apart! But for myself, I mean the last thing I ever think about is the fact that I somehow got this diabetes a couple of years ago-it has absolutely no influence on my daily life" (i3, male, 58y, T2D)

Even if most of the interviewees with T2D ignored diabetes in the context of COVID-19, there was a tendency for those with T2D who also had other reasons for being at increased risk to take it seriously. In these cases, however, the main reason for taking the risk seriously was rarely the diabetes:

"I definitely worry. It doesn't sound pleasant at all-even the mild cases. I mean, with my age, 73, and hypertension and now diabetes, I'm in the risk group. So yeah, I'm a bit worried." (i8, male, $73 \mathrm{y}, \mathrm{T} 2 \mathrm{D})$

The general tendency among the interviewees with T2D, however, was to not take it particularly seriously. Many of them told us that their relatives worried much more than they themselves did:

"Our kids believe that I need to be extra careful. They tell me on a regular basis that now I'm in the danger group or whatever it's called? Risk group. They worry about me more than I do myself" (i10, male, 69y, T2D)

Even when the media placed people with T2D firmly in the risk group and the people close to them tended to express worries about the relation between T2D and COVID-19, the interviewees with T2D tended to significantly downplay the severity of the situation, basically observing themselves as not belonging to a particular risk group. The major reason for this seemed to be that they did not identify with their disease in the first place, and therefore their self-understanding was not strongly connected with them having a chronic disease.

\subsection{Changes in Relational Everyday Life}

How people deal with abrupt changes and/or increased complexity is deeply dependent on their individual ability to relate to communication in social systems. What happens when the basic aspects of relational everyday life change from one day to the next? How do people with diabetes then cope with general meaning-making and basic self-observation?

"It really is kinda unsettling when everyone around you suddenly goes: You really have to take special care now'" (i16, male, 68y, T2D)

Even though many of the interviewees with T2D had done their best to ignore and dismiss their heightened risk, they were also the ones who were most frustrated about the discrepancies between their view of themselves as healthy and not at risk and how the environment tended to view them as being at risk. They were basically observing the environment's observations of them as out of tune with 
the meaning they had created through their own observation. This created a sense of meaning-crisis in several of them, and they seemed especially vulnerable to these changes in relational and public perceptions. Immediately before her interview, one female interviewee with T2D had just spoken with her son about this, and the conversation had changed the way she observed herself in relation to risk:

"This happened just an hour ago. I had to concede, and I just thought: 'Oh my god, he's right.' I mean, we have to take care of ourselves in this vulnerable situation we're in. I am in the risk group. So, it's actually fantastic that our son thinks about it. It warms my heart that we have children who think like that" (i4, female, 71y, T2D)

Being able to meaningfully observe the worries and underlying affection of close relations seemed to create the basis for changes in both self-observations and risk perceptions.

Observing yourself being observed by the media (and society as a whole) as vulnerable or at risk had left a strong impression on many of the interviewees:

"This is just so difficult to change. If you have a population that possesses a piece of knowledge that they believe is true, then it really takes so much to change that in any way ... So, no matter how much empathy or love you have for a given disease population, you will, in some way be affected by what the rest of society thinks about this disease ... And I guess we're just really unlucky in that respect" (i2, female, 26y, T1D)

This relationship with the rest of society was a burden, especially for the interviewees with T1D. They often felt a general lack of knowledge about what diabetes is and does. Further, they felt that this lack of knowledge strongly affected how they were observed by society and, consequently, how they ultimately observed themselves. This situation of observing close and distant relations in the complex network of social systems was one of reducing complexity by deciding or knowing where to observe, so as to continually be able to create a sense of meaning that corresponds with the sense of self they were simultaneously creating through their perpetual self-observations.

In order to create this sense of meaning, many of them had placed a great deal of value on their peer relationships:

"Yesterday I was chatting with some friends who also have diabetes and I just thought that I'm so lucky to have them, because, just imagine all the people with diabetes who don't know anyone else with diabetes ... They're just extra alone right now and that must be really hard." (i2, female, 26y, T1D)

Creating communicative meaning by seeking out well-known and well-established relationships in times of crisis and insecurity was a way for especially the younger interviewees with T1D to reduce communicative complexity. This appeared to have created a situation with stronger internal identification and feelings of belonging in these groups, while also weakening external identification and creating a feeling of being left out or forgotten.

The lockdown and the perception of being at risk had also affected routines and the everyday understanding of roles in close relationships for the interviewees with T1D:

"I do understand that it's frustrating to live with a diabetic right now, because there's really not a lot I can help him with. Normally we're like an old married couple with certain things each of us does more than the other. We've divided the chores and that's just completely [obscenity] up right now. I don't do any shopping, I don't see any friends, I can't go to work and since I'm working freelance, I make less money. I can't go to uni to meet with my study partner 'cause I just think it's too dangerous." (i6, female, 27y, T1D)

This feeling of having your everyday life turned upside down was something all the interviewees with T1D described. For many of them, this was linked to the frustration of not having received any definite risk assessments from the authorities, which had turned into a negative spiral of regrets, doubts and fears that life as they knew it was falling apart. They felt this put great pressure on their 
important relations and, as the young woman in the above quote clearly described, these doubts had caused them to constantly observe how they were being observed by their loved ones. This made them feel like a burden, which again fed the negative spiral with more and more negative self-observation.

\section{Discussion}

In our study, we have identified three highly interdependent themes of relevance to how people with diabetes navigate living with the disease during the COVID-19 lockdown and how this affects their sense of self and of being a part of society. These themes are all strongly related to structures of observation, or to be more precise, structures of observing observation [22]. The interviewees all mentioned how they had tried to make sense of communication from health authorities, mass media, peer groups and family members, while at the same time trying to make sense of the way these communicative sources were observing people with diabetes as belonging to risk groups and/or being vulnerable. This mutual process of observations is one that is ongoing at any time and in any society, but as the results of our study show, the challenges of having to create and re-create meaning internally (psychological systems) and externally (social systems) can be especially demanding at times when very basic elements of meaning, and of how we are able to observe each other, are changing dramatically. These findings add significant new knowledge about when and how to apply theories about communicative social systems and maybe most of all highlights the potentials in applying the concept of observation to how vulnerable groups react to societal developments [21-23].

These experiences of living in a society that is locked down illuminate the mechanisms of psychological systems trying to create meaning based on observations of social systems. The fact that normal everyday interactions with friends, colleagues, family members and the healthcare professionals suddenly are extremely limited, creates a new and unknown situation, where this meaning-making is severely challenged-causing insecurities and the personal need for complexity-reducing health- and risk information.

When traditional structures for observing are no longer operational, the meaning-making process of creating expectational patterns as a way of reducing complexities in daily life is becoming a daily struggle for the people with diabetes, not knowing what the future brings or how to create meaning based on the observed communication. Most significantly this lack of meaning regards being placed in a group defined and observed as vulnerable or at risk.

In the study, we saw significant differences between persons with T1D and those with T2D in regards how they experience themselves, their supportive relations and their diabetes in relation to COVID-19 during the lockdown, but we have also shown the general (both T1D and T2D) ambivalence associated with being observed as belonging to a vulnerable group. Whereas the interviewees with diabetes created meaning out of the situation in a multitude of ways, they all responded to being labelled as persons at risk. Having to suddenly observe themselves being observed in a very different way than before had forced them to adapt to this observation by adjusting how they observe themselves. This significant finding has not been reported in other studies so far and is, therefore, a valuable contribution to the existing research in COVID-19 and diabetes.

All 20 participants had different disease trajectories that could potentially impact their reaction to COVID-19 and the lockdown. Apart from the significant differences between the two main types of diabetes, it could be assumed that an aspect like years since diagnosis would play a role in how people with diabetes manage their disease in a time of change and insecurities. We did, however, not find strong indications of that.

Coming from a different theoretical angle than Luhmann did, philosopher Ian Hacking argued that such labels may have 'looping effects' in so far as people change their behaviours and self-understandings in response to the categories in which they are placed [27]. In the present case, the categorization of people with diabetes as a vulnerable group appears to have had consequences for their behaviours, in terms of diabetes management and social isolation, as well as for the extent to which they identified with their disease. The effects become 'looping' because these changes reflect 
back on their vulnerability by transforming the very conditions that made them vulnerable in the first place. Hacking's theory supports our findings, and further research might benefit from an analytical strategy that combines Hacking's and Luhmann's respective approaches.

Our findings have important implications for health communication in general and risk communication in particular. They show that people may respond in a multitude of ways to communications issued by health authorities and that their meaning-making is shaped by, and shapes, their self-observations, risk perceptions and close relational environments. This may call for more targeted communication strategies as well as increased use of peer support that can help people create meaning within their own environments. This is relevant to both micro-level communications with the diabetes clinic or the GP and macro-level communication on the societal level. In fact, this split-level communication strategy is especially pertinent to people with diabetes, as they are exposed to the massive amount of risk communication dealing with COVID-19 risk on a more general level and need diabetes-specific clarifications from specialist sources.

As we wished to study people with diabetes and their immediate reactions to the COVID-19 lockdown, we had to plan and perform the interview study within a very short period of time. This meant that things were done more quickly than would normally be the case. We focused on eliminating rushed mistakes by keeping everything as simple as possible, though without compromising our research standards. We decided to keep the interview guide very basic and open, as we did not have sufficient background literature to target specific pre-themes or pre-understandings. If we had conducted the study two or four months later, we would have been able to make it more specific because there would have been more time for preparation and more knowledge about COVID-19 and societal reactions in general. On the whole, however, we are satisfied with the richness and depth of the data.

The external validity or transferability of the study at hand is somewhat difficult to assess given the novelty of the societal situation studied. To what extent our findings can be applied beyond the contexts in which the studies were done, and how a more optimal sample might be constructed in order to make the findings more transferable is indeed rather difficult to say. Regarding recruitment, we decided to include people with different types of diabetes in the same study. We did this because we did not know what we would find. Although the two types of diabetes (and the daily tasks of managing the diseases) are significantly different, we did not know whether this would transfer to reactions to the risks associated with COVID-19. Another reason for including both types was that the general risk information issued by health authorities in the early stages of the pandemic was generally not specific to diabetes type. In retrospect, the differences between the two groups helped highlight the main findings of our study. The reactions to COVID-19 and the lockdown, however, were so different that future studies could benefit from a more specific focus on the characteristics of the two main types of diabetes. This would give us a more in-depth understanding of some of the elements that were specific to T1D and T2D.

Another aspect of the transferability is the choice of theory. Working with the theory in our study makes it clear that the actual choice of theory strongly affects the degree of transferability. This is very much in line with Rasmussen's [24] description of how, within radical hermeneutics, there is a constant constructivist awareness of the relationship between theory and the construction of the environment observed. Employing a theory that focuses on complexity makes the findings more flexible and adaptable to different contexts and settings, which is especially relevant in a situation, where we are studying a novel and previously unknown societal phenomenon.

Author Contributions: Conceptualization, D.G., J.M. and M.H.R.; Formal analysis, D.G.; Investigation, D.G. and J.M.; Methodology, D.G., J.M. and M.H.R.; Writing_original draft, D.G.; Writing—review \& editing, J.M. and M.H.R. All authors have read and agreed to the published version of the manuscript.

Funding: This research received no external funding.

Conflicts of Interest: The authors declare no conflict of interest. 


\section{References}

1. Cucinotta, D.; Vanelli, M. WHO Declares COVID-19 a Pandemic. Acta Biomed. 2020, 91, 157-160.

2. Rothan, H.A.; Byrareddy, S.N. The epidemiology and pathogenesis of coronavirus disease (COVID-19) outbreak. J. Autoimmun. 2020, 102433. [CrossRef] [PubMed]

3. Diabetesforeningen [Danish Diabetes Association]. Sundhedsstyrrelsen: Alle med Diabetes er i Risikogruppen [Danish Health Authority: All People with Diabetes Are at Risk]. 2020. Available online: https://diabetes.dk/aktuelt/nyheder/nyhedsarkiv/2020/sundhedsstyrelsen-alle-med-diabetes-er-i-risikogr uppen.aspx (accessed on 18 July 2020).

4. Kumar, A.; Arora, A.; Sharma, P.; Anikhindi, S.A.; Bansal, N.; Singla, V.; Khare, S.; Srivastava, A. Is diabetes mellitus associated with mortality and severity of COVID-19? A meta-analysis. Diabetes Metab. Syndr. 2020, 14, 535-545. [CrossRef] [PubMed]

5. Wang, X.; Wang, S.; Sun, L.; Qin, G. Prevalence of diabetes mellitus in 2019 novel coronavirus: A meta-analysis. Diabetes Res. Clin. Pract. 2020, 164, 108200. [CrossRef] [PubMed]

6. Fadini, G.P.; Morieri, M.L.; Longato, E.; Avogaro, A. Prevalence and impact of diabetes among people infected with SARS-CoV-2. J. Endocrinol. Investig. 2020, 43, 867-869. [CrossRef] [PubMed]

7. Huang, I.; Lim, M.A.; Pranata, R. Diabetes mellitus is associated with increased mortality and severity of disease in COVID-19 pneumonia-A systematic review, meta-analysis, and meta-regression. Diabetes Metab. Syndr. 2020, 14, 395-403. [CrossRef]

8. Cuschieri, S.; Grech, S. COVID-19 and diabetes: The why, the what and the how. J. Diabetes Complicat. 2020, 34, 107637. [CrossRef]

9. Gupta, R.; Hussain, A.; Misra, A. Diabetes and COVID-19: Evidence, current status and unanswered research questions. Eur. J. Clin. Nutr. 2020, 74, 864-870. [CrossRef]

10. Katulanda, P.; Dissanayake, H.A.; Ranathunga, I.; Ratnasamy, V.; Wijewickrama, P.S.A.; Yogendranathan, N.; Gamage, K.K.K.; de Silva, N.L.; Sumanatilleke, M.; Somasundaram, N.P.; et al. Prevention and management of COVID-19 among patients with diabetes: An appraisal of the literature. Diabetologia 2020, 1-13. [CrossRef]

11. Singh, A.K.; Gupta, R.; Ghosh, A.; Misra, A. Diabetes in COVID-19: Prevalence, pathophysiology, prognosis and practical considerations. Diabetes Metab. Syndr. 2020, 14, 303-310. [CrossRef]

12. Targher, G.; Mantovani, A.; Wang, X.B.; Yan, H.D.; Sun, Q.F.; Pan, K.H.; Byrne, C.D.; Zheng, K.I.; Chen, Y.P.; Eslam, M.; et al. Patients with diabetes are at higher risk for severe illness from COVID-19. Diabetes Metab. 2020. [CrossRef]

13. Guo, W.; Li, M.; Dong, Y.; Zhou, H.; Zhang, Z.; Tian, C.; Qin, R.; Wang, H.; Shen, Y.; Du, K.; et al. Diabetes is a risk factor for the progression and prognosis of COVID-19. Diabetes Metab. Res. Rev. 2020, e3319. [CrossRef] [PubMed]

14. Zhou, F.; Yu, T.; Du, R.; Fan, G.; Liu, Y.; Liu, Z.; Xiang, J.; Wang, Y.; Song, B.; Gu, X.; et al. Clinical course and risk factors for mortality of adult inpatients with COVID-19 in Wuhan, China: A retrospective cohort study. Lancet 2020, 395, 1054-1062. [CrossRef]

15. Singh, A.K.; Singh, R. Does poor glucose control increase the severity and mortality in patients with diabetes and COVID-19? Diabetes Metab. Syndr. 2020, 14, 725-727. [CrossRef] [PubMed]

16. Zhang, Y.; Li, H.; Zhang, J.; Cao, Y.; Zhao, X.; Yu, N.; Gao, Y.; Ma, J.; Zhang, H.; Zhang, J.; et al. The clinical characteristics and outcomes of diabetes mellitus and secondary hyperglycaemia patients with coronavirus disease 2019: A single-center, retrospective, observational study in Wuhan. Diabetes Obes. Metab. 2020, 22, 1443-1454. [CrossRef]

17. Adhikari, S.P.; Meng, S.; Wu, Y.J.; Mao, Y.P.; Ye, R.X.; Wang, Q.Z.; Sun, C.; Sylvia, S.; Rozelle, S.; Raat, H.; et al. Epidemiology, causes, clinical manifestation and diagnosis, prevention and control of coronavirus disease (COVID-19) during the early outbreak period: A scoping review. Infect. Dis. Poverty 2020, 9, 29. [CrossRef]

18. Mainous, A.G. A towering babel of risk information in the COVID-19 pandemic: Trust and credibility in risk perception and positive public health behaviors. Fam. Med. 2020, 52, 317-319. [CrossRef] [PubMed]

19. Statsministeriet [The Prime Minister's Office]. Pressemøde om COVID-19 den 11. marts 2020 [Press Conference on COVID-19 the 11th of March 2020]. Available online: https://www.stm.dk/_p_14920.html (accessed on 22 July 2020).

20. Rudnick, A. Social, psychological and philosophical reflections on pandemics and beyond. Societies 2020, 10, 42. [CrossRef] 
21. Luhmann, N. Essays on Self-Reference; Columbia University Press: New York, NY, USA, 1990.

22. Luhmann, N. Social Systems; Stanford University Press: Palo Alto, CA, USA, 1995.

23. Luhmann, N. Theories of Distinction; Stanford University Press: Palo Alto, CA, USA, 2002.

24. Rasmussen, J. Textual interpretation and complexity-Radical hermeneutics. Nord. Pedagog. 2004, 24, 177-194.

25. Carlsson, S. Environmental (Lifestyle) risk factors for LADA. Curr. Diabetes Rev. 2019, 15, 178. [CrossRef]

26. Malterud, K.; Siersma, V.D.; Guassora, A.D. Sample size in qualitative interview studies: Guided by information power. Qual. Health Res. 2016, 26, 1753-1760. [CrossRef] [PubMed]

27. Hacking, I. The looping effects of human kinds. In Causal Cognition. A Multidisciplinary Debate; Sperber, D., Ed.; Clarendon Press: Oxford, UK, 1995; pp. 351-394.

C 2020 by the authors. Licensee MDPI, Basel, Switzerland. This article is an open access article distributed under the terms and conditions of the Creative Commons Attribution (CC BY) license (http://creativecommons.org/licenses/by/4.0/). 